\title{
Exploring the potential of endophytes in agriculture: a minireview
}

\section{Introduction}

Endophytes are the group of microorganisms that live inside host microenvironment, receive protection from environmental stresses, face lesser competition from other microbes and have greater access to nutrients. ${ }^{1}$ Since endophytes interact closer to plant than rhizosphere and phyllosphere bacteria, their effects to the plants may be direct and intense. Plant intercellular spaces are good sites for endophyte multiplication because of the richness of nutrients like potassium, calcium, sulfur, phosphorous and chlorine and carbohydrates, ${ }^{2,3}$ various amino acids and organic acids. ${ }^{4}$ Endobacteria directly benefit plants by stimulating their growth and/or indirectly through a reduction in the incidence of plant disease. Endophytes improve seedling performance and survival by providing resistance against insects and nematodes resistance and drought, improving nitrogen assimilation thus yielding higher seed set. ${ }^{5}$

\section{How endophytes benefit plants?}

Very little is known about the endophyte directed mechanisms of plant growth promotion. ${ }^{6}$ Endophytes mediate plant growth promotion through direct or indirect mechanisms. Since endophytes start their journey as rhizosphere bacteria, it is assumable that they may retain their attributes inside plant. Their mechanisms of benefit seem related to rhizosphere bacteria because most of endophytes can be cultured and can survive outside host in rhizosphere.

\section{Directly beneficial mechanisms}

Endophyte can directly benefit plants by providing antimicrobial metabolites, ${ }^{7}$ insecticidal by-products, ${ }^{8}$ iron chelators, ${ }^{9}$ phosphate solubilizing compounds and nitrogen fixing abilities. ${ }^{10}$ In addition, endophytes influence plant growth through production of phytohormones, siderophores, induced systemic tolerance through 1-aminocyclopropane-1-carboxylase deaminase production, induced systemic resistance and antagonism. Also, several sulfur oxidizing endophytes are known which oxidize elemental sulfur into sulfate to be used by plants. ${ }^{11}$ Moreover, endophytes are prolific sources of phytochemicals ${ }^{12}$ which impede plant pathogens. ${ }^{13,14}$ Endophytes are a good source of biologically active secondary metabolite ${ }^{15,16}$ and contribute in plant metabolite production. ${ }^{15}$ Some of the important and well-cited mechanisms are discussed below. These mechanisms may induce direct or indirect benefit to plant.

Phytohormone production: Plant growth promotion by endophytes through phytohormone production is perhaps the well-agreed method which causes morphological and structural changes in the plant. Because of these qualities, endophytes find their application in agricultural system for sustainable agriculture. ${ }^{17}$ The mechanism of phytohormones production by endophytes in host plants is similar to that of plant growth promoting rhizobacteria. They enhance the growth of non-legumes by improving their growth through the production of indole acetic acid, ${ }^{18,19}$ gibberellic acid, ${ }^{19}$ ethylene ${ }^{20}$ and auxins. ${ }^{1}$

Nitrogen fixation: Nitrogen supply is the most limiting nutritional factor for plant growth. Plants cannot reduce atmospheric $\mathrm{N}$ and therefore require an external fixed $\mathrm{N}$ supply. Biological $\mathrm{N}$ fixation
Volume 6 Issue 4 - 2017

\author{
Anurag Yadav,' Kusum Yadav ${ }^{2}$ \\ 'Department of Microbiology, College of Basic Science \& \\ Humanities, SD Agricultural University, India \\ ${ }^{2}$ Department of Biochemistry, University of Lucknow, India
}

Correspondence: Anurag Yadav, Department of Microbiology, College of Basic Science \& Humanities, SD Agricultural University, SK Nagar, Gujarat, India, Tel +9 I-972-47 I-47I0, Email anuragyadav123@gmail.com

Received: September 08, 2016 | Published: February 2I, 2017

finds immense potential as an alternate to chemical fertilizers. Several symbiotic prokaryotic endophytes which have the ability to fix atmospheric nitrogen have potential in agriculture. Diazotrophic endophytes can directly transport nitrogen to plants. Nitrogen fixing free-living endophytic bacteria have been the focus of study since past few decades. ${ }^{21}$ The most famous and well-studied legume-Rhizobium symbiosis is still the subject of worldwide research which is directed towards enhancing the N2 fixation efficiency through plant and bacterial genome manipulation.

Phosphate solubilization: Apart from N2 fixation, several endophytes release organic acids into the soil which solubilize the phosphate complexes and convert it into ortho-phosphate for plant up-take and utilization. Although phosphorous exist abundantly in soils, but the majority of it remains unavailable as an insoluble form. ${ }^{22}$ Several literature findings discuss the role of endophytes as phosphate solubilizer. For e.g. endophyte Pantoea spp associated with root nodule of peanut have been shown to possess phosphate solubilizing activity.

Siderophore production: Some endophytes produce small molecular compounds called siderophores which are iron chelating compounds that can avail iron to plants and deprive pathogen of iron..$^{23}$ Out of the range of siderophore produced by endophytes one with biocontrol properties are catacholate, hydroxymate and/or phenolate types. ${ }^{24}$ Also, siderophores specifically help iron deficient plant in fixing nitrogen since diazotrophs require $\mathrm{Fe}++$ and Mo factors for nitrogenase synthesis and functioning. ${ }^{25}$

There is a plethora of literature to support the insecticidal effect of endophytes. ${ }^{26}$ Some endophytes induce thickening of the endodermal cell wall, which reduce the chances of pest penetration in stele. ${ }^{27}$ Others intoxicate insects by secreting secondary metabolites. Toxicant metabolites like pyrrolopyrazine alkaloid peramine, ${ }^{28}$ ergot alkaloid ergovaline ${ }^{28}$ and pyrrolizidine loline alkaloids ${ }^{29}$ have been reported in several studies.

\section{Indirectly beneficial mechanisms}

Plants cope with a series of unfavourable environmental and biotic 
stresses like drought, cold, hypersaline condition, or pathogenesis Endophytes help plant to overcome such stresses through some indirect mechanisms. For e.g. the mechanism of induced systemic resistance (ISR) states that some endophytes which may have evolved from plant pathogens could induce plant defence responses like pathogens. Some of the known mechanisms are discussed below.

Bioremediation: Bioremediation refer to the methods of removing pollutants from the environment. Plants secrete some toxic metabolites, which it is unable to neutralize. The study of microbial metabolism and the factors that influence the process is the key for understanding bioremediation. Better understanding of underlying microbial processes will allow us to alter the mechanism by molecular tools and thus may help in enhancing effectiveness of bioremediation. Endophytes can help in bioremediation through several means. They reduce heavy metal stress to plants, ${ }^{30}$ degrade toxic components ${ }^{3}$ and toxic metabolites secreted by plants ${ }^{32}$ remove greenhouse gases from $a_{i r^{33}}$ and control pest growth on plants. ${ }^{26}$ Several methods are available to induce effective bioremediation and some more cost effective techniques are in the process of development.

Phytoremediation: Endophytes help in phytoremediation by enhancing plant growth, decreasing metal phytotoxicity and by affecting metal translocation and accumulation. Plant-endophyte partnership can be harnessed to remediate wastelands and ground water since endophytes provide plants with required degradation pathways and metabolic abilities for enhancing degradation and reducing phytotoxicity. ${ }^{34}$ They boost phytoremediation and promote plant growth through nitrogen fixation, mineral solubilization, phytohormones production, siderophore production, using 1-aminocyclopropane-1-carboxylic acid as $\mathrm{N}$ source and by nutrient transformation..$^{33}$

Endobacteria help in facilitating photoextration of heavy metals. ${ }^{3.5}$ Several successful trials of heavy metal removal employing endophytic bacteria along with plants have been conducted. Endophytes are also helpful in polyaromatic hydrocarbon degradation (PAH) Phytoremediation of oily soils using rhizosphere bacteria seems to be the cost-effective and eco-friendly way of cleaning ${ }^{36}$ and remediating polyaromatic hydrocarbon based soil contaminants. ${ }^{37}$ Plants degrade PAHs by enhancing microbial density in rhizosphere and endosphere to enhance catalysis of atmospheric oxygen into aliphatic or aromatic hydrocarbons, producing corresponding alcohols. ${ }^{36}$ Such type of phyto remediating microflora might be naturally present in the plants growing in chronically contaminated sites. For e.g. halophytic plants like Halonemum strobilaceum, inhabiting the coastal regions of the Arabian Gulf possess hydrocarbon utilizing microflora ${ }^{38}$ and thus may reduce oil load on costal area.

Biocontrol: Biocontrol refers to the eco-friendly way of reducing plant pathogens that may cause damage to agriculture crops through natural antagonists. ${ }^{39}$ Most of the antagonistic rhizosphere bacteria are known biological control agents and belong to genera Bacillus and Pseudomonas. However, their use as an effective biocontrol agent is limited by their inability to survive and colonize nonnative microclimates. Endophyte, on the other hand, has a survival advantage over rhizosphere bacteria since they live in protected microenvironment of the host. Most of the antagonistic endophytes are Gram-negative and members of the family pseudomonaceae; the entire group of fluorescent pseudomonads has evolved as biological control agent (BCA).

Like rhizosphere bacteria endophytes also reduce plant pathogen severity by competing for nutrition in the same ecological niche and by producing chemical substances harmful to plant pathogens, thus affecting plant pathogens directly, indirectly or ecologically. ${ }^{40}$ Some endophytes produce antibiotics in the rhizosphere, which controls growth of harmful bacteria. ${ }^{41}$ Antibiotics like ecomycins, pseudomycins, munumbicins and xiamycins ${ }^{42}$ with antibacterial properties have been isolated from endophytes of several plants. Other than antibiotics, endophytes produce a variety of metabolites, which are applicable in agrochemicals and pharmaceuticals. These metabolic products ${ }^{42}$ play a crucial role in shaping the endosphere microflora. The metabolites like flavonoids and flavones, which are plant-signalling molecules with antimicrobial properties, are produced as signalling response of microbial adhesion to root surface (phytoalexins). Endophytes also reduce the impact of virus infection on host, suggesting their role in induction of host response. Plant viral diseases are often difficult to control directly, instead they are indirectly controlled by killing the 'pests' involved as vector in disease. ${ }^{43}$ Involvement of selective endophytes that can reduce the intensity of viral disease will help us in reducing our dependency on chemical pesticides.

Biocontrol mechanism: Endophyts possess a range of biocontrol mechanisms to counteract plant pathogens and insects. As a measure of defence, plant secretes signalling molecules against pathogens in endosphere. Plants tackle differently with pathogenic, associative, symbiotic, or neutralistic microorganism through signalling molecules. ${ }^{44}$ Association of endophytes help plants to better interact with other organisms.

As a means of biocontrol, Endophytes can elicit ISR in plants, causing disease severity reduction and plant stress tolerance improvement. ${ }^{45}$ Some endophytic bacteria might actually elicit plant defence although they are not spatially separated from the pathogen. ${ }^{46}$ Endophytes may also augment the defence against herbivores and insects. ${ }^{47,48}$ There are cascades of physiological steps in signal transduction that lead to induced resistance to plants. It has been found that B. pumilus SE34 triggers ISR in a sequence of steps starting with elaboration of structural barriers, production of toxic substances (e.g. phenolics and phytoalexinc), accumulation of molecules (e.g. chitinase) and hydrolytic enzymes (e.g. $\beta-1,3-$ glucanases), which contribute in releasing oligosaccharides that results in stimulating other defence reactions. ${ }^{14}$ The ISR is induced to restrict of pathogens to outer plant root corte ${ }^{49}$ by increasing host cell wall density to restrict potential pathogen penetration, ${ }^{14}$ which is activated by expression of pathogenesis-related (PR) proteins, (chitinase and b-1,3-glucanase) and defence-related proteins, (peroxidase, polyphenol oxidase, phenylalanine ammonia-lyase) and phenolic compounds..$^{50}$ Endophytes also induce ISR to plants to fight against viral pathogens. The reports show induction of ISR in tomato plants by endophytes, Bacillus subtilis IN937b, B. pumilus SE34, and B. amyloliquefaciens IN937a against cucumber mosaic cucumovirus (CMV) ${ }^{51}$ Several endophytes cause ISR in plants against banana bunchy top virus. ${ }^{50}$ Also, the common endophyte genus Paenibacillus is known to produce soluble and volatile metabolites that may inhibit the pathogen growth. ${ }^{39}$ Moreover, endophytes protect plants from root pathogens by preparing biofilm around the roots. ${ }^{39}$ The experiment on tall fescue (Festuca arundinacea Schreb.) shows that endophyte infection enhances production volatile compound, monoterpene $\beta$-ocimene. Also other monoterpenes such as (E,Z)-allo-ocimene, limonene, myrcene, linalool and other compounds like methyl salicylate, indole and nonanal are produced in increased amounts due to endophyte infection. ${ }^{52}$ 
Plant stress tolerance: In nature, plants face a variety of stresses and react by modifying their own metabolism to get tolerant. The beneficial endophytic bacteria may mediate plant adaptation to the environmental stress. ${ }^{53}$ PGPR endobacteria alleviate plant stress due to temperature, drought, heavy metal accumulation and solar ultraviolet-B radiation $(280-315 \mathrm{~nm}) .^{54}$ It has been shown that endophyte enhance cold tolerance by altering photosynthetic activity and metabolism of carbohydrates resulting in deposition of cold stress related metabolites like starch, proline and phenolics. ${ }^{55,56}$ Endophytes show similar effects due to drought stress. ${ }^{57}$ Likewise, plants deal with salinity stress by accumulating endophyte mediated glycine betain-like compounds. ${ }^{58}$ Also, abscisic acid (ABA) secreted by Azospirillum lipoferum alleviate water-stress of maize plants. It has been hypothesized that ABA signals the plant to resist water deficiency by controlling stomata closure to reduce water loss. ${ }^{59}$ Another stress type, salinity, hinders crop yield of many plants through ethylene biosynthesis ${ }^{60}$ that inhibits root growth. Reports show that ethylene level, which is a measure of plant stress, can be reduced by some plant growth promoting endobacteria by secreting ACC deaminase. ${ }^{61}$

Also, the endophytes help host competitiveness towards pathogens by some unknown mechanisms, which is independent of increased growth. ${ }^{62}$ These mechanisms may involve increased production of allelochemicals; increased plant vigor and seed yield, ${ }^{63}$ increased tiller numbers and leaf elongation rate and alteration of root architecture. ${ }^{64}$

\section{Conclusions and future outlook}

The depth of scientific understanding about endophytes is largely in its infancy with research published in the literature is either scarce or not fully understood. ${ }^{65}$ Endophyte study, which previously remained hindered due non-cultivability of some endophytes is now benefitting from culture independent methods of bacterial identification. The entophytes must possess following attributes for agricultural exploitation. They

i. must not induce plant disease,

ii. should be capable to spread inside plant parts,

iii. should be culturable and,

iv. must colonize plant parts naturally obligately with species specificness. ${ }^{66}$ There is a strong need to search for novel entophyte strains with as many desirable characters for enhancing the crop yield.

Newer approaches of exploration, like search for novel endophytes or endophyte gene alteration are on the horizon of replacing the need for host specific studies. Instead, novel endophytes can be screened for desirable traits from plants growing under extreme environments. The alternative approach of gene manipulation can equip host plants with new traits like herbicide resistance, phytoremediation etc. which could more suitably regulate metabolism.

No microbial technology can be considered successful until its commercial viability is proved. Endophyte specificity with the host plant is the major hurdle in its large-scale production. The detailed host specific population dynamics study of endophyte is required before starting the bulk production, which requires technology based advances in research. Efforts also require formulation of plant specific inoculum doses of endophytes. The optimized host specific inoculum dosage will reduce cost of bulk inoculum production and application and thus may enhance productivity. Enhanced productivity will help to reduce our dependency on chemical fertilizers, pesticides and fungicides. Future discovery of pesticides with synergistic effect on endophyte bioinoculant may be able to control the range of pathogens. The development of sprayable endophytes for use along with chemical pesticides will pave the way for commercial pesticide development for effective integrated pest management.

\section{Acknowledgements}

None.

\section{Conflict of interest}

The author declares no conflict of interest.

\section{References}

1. Dutta D. Endophytes:exploitation as a tool in plant protection. Brazilian Archives of Biology and Technology. 2014;57:621-629.

2. Madore M, Webb JA. Leaf free space analysis and vein loading in Cucurbita pepo. Canadian Journal of Botany. 1981;59(12):2550-2557.

3. Canny M, McCully M. The xylem sap of maize roots:its collection, composition and formation. Functional Plant Biology. 1988;15(4):557-566.

4. Canny M, Huang C. What is in the intercellular spaces of roots? Evidence from the cryo $\square$ analytical $\square$ scanning electron microscope. Physiologia Plantarum. 1993;87(4):561-568.

5. Fescue T. A Review of the Agronomic characteristics of endophyte-free and endophyte-infected. Applied Agricultural Research. 1990;5(3):188194.

6. Hardoim PR, van Overbeek LS, Elsas JD. Properties of bacterial endophytes and their proposed role in plant growth. Trends in Microbiology. 2008;16(10):463-471.

7. Pinheiro EA, Josiwander Carvalho M, André O Feitosa, et al. Chemical constituents of Aspergillus sp EJC08 isolated as endophyte from Bauhinia guianensis and their antimicrobial activity. An Acad Bras Cienc. 2013;85(4):1247-1253.

8. Azevedo JL, Walter Maccheron, José Odair Pereira, et al. Endophytic microorganisms:a review on insect control and recent advances on tropical plants. EJB Electronic Journal of Biotechnology. 2000;3(1):1-26

9. Long H, Schmidt D, Baldwin I. Native bacterial endophytes promote host growth in a species-specific manner; phytohormone manipulations do not result in common growth responses. PLoS One. 2008;3(7):e2702.

10. Knoth JL. Biological nitrogen fixation and biomass accumulation within poplar clones as a result of inoculations with diazotrophic endophyte consortia. New Phytol. 2014;201(2):599-609.

11. Banerjee MR, Yesmin L. Sulfur-oxidizing plant growth promoting rhizobacteria for enhanced canola performance. Google Patents. 2009.

12. Nisa H. Fungal endophytes as prolific source of phytochemicals and other bioactive natural products:A review. Microbial Pathogenesis. 2015;82:50-59.

13. Chen B. A bacterial endophyte from banana:its isolation, identification, activity to Fusarium Wilt and PGPR effect to banana seedlings. Microbiology/Weishengwuxue Tongbao. 2011;38(2):199-205.

14. Benhamou N, Kloepper JW, Tuzun S. Induction of resistance against Fusarium wilt of tomato by combination of chitosan with an endophytic bacterial strain:ultrastructure and cytochemistry of the host response. Planta. 1998;204(2):153-168.

15. Brader G. Metabolic potential of endophytic bacteria. Current Opinion in Biotechnology. 2014;27:30-37. 
16. Schulz B, Christine Boyle, Siegfried Draeger, et al. Endophytic fungi:a source of novel biologically active secondary metabolites. Mycological Research. 2002;106(9):996-1004

17. Sturz AV, Christie BR, Nowak J. Bacterial endophytes:Potential role in developing sustainable systems of crop production. Critical Reviews in Plant Sciences. 2000;19(1):1-30.

18. Patel MV, Patel RK. Indole-3-acetic acid (IAA) production by endophytic bacteria isolated from saline dessert, the Little Rann of Kutch CIBTech J Microbiol. 2014;3:17-28.

19. Khan AL. Bacterial endophyte Sphingomonas sp. LK11 produces gibberellins and IAA and promotes tomato plant growth. $J$ Microbiol. 2014;52(8):689-695

20. Kang JW, Khan Z, Doty SL. Biodegradation of trichloroethylene by an endophyte of hybrid poplar. Appl Environ Microbiol. 2012;78(9):3504 3507.

21. Reis V. Burkholderia tropica sp. nov., a novel nitrogen-fixing, plant-associated bacterium. International Journal of Systematic and Evolutionary Microbiology. 2004;54(6):2155-2162.

22. Miller SH. Biochemical and genomic comparison of inorganic phosphate solubilization in Pseudomonas species. Environmental microbiology reports. 2010;2(3):403-411.

23. Compant S. Use of plant growth-promoting bacteria for biocontrol of plant diseases:principles, mechanisms of action, and future prospects. Applied and environmental microbiology. 2005;71(9):4951-4959.

24. Rajkumar M. Potential of siderophore-producing bacteria for improving heavy metal phytoextraction. Trends in Biotechnology. 2010;28(3):142149.

25. Kraepiel A. Multiple roles of siderophores in free-living nitrogen-fixing bacteria. Biometals. 2009;22(4):573-581.

26. Azevedo JL. Endophytic microorganisms:a review on insect control and recent advances on tropical plants. Electronic Journal of Biotechnology 2000;3(1)

27. Gwinn KD, Bernard EC. Interactions of endophyte infected grasses with the nematodes Meloidogyn marylandi and Pratylenchus scribneri, in Proceeding of 2nd international symposium Acremonium /grass interact Plenary Papers: Plamerston North; 1993.

28. Siegel M. Fungal endophyte-infected grasses:alkaloid accumulation and aphid response. Journal of chemical ecology. 1990;16(12):3301-3315.

29. Wilkinson $\mathrm{HH}$. Contribution of fungal loline alkaloids to protection from aphids in a grass-endophyte mutualism. Molecular Plant-Microbe Interactions. 2000;13(10):1027-1033.

30. Zhang, X, Li C, Nan Z. Effects of cadmium stress on seed germination and seedling growth of Elymus dahuricus infected with the Neotyphodium endophyte. Sci China Life Sci. 2012;55(9):793-799.

31. Han JI. Complete genome sequence of the metabolically versatile plant growth-promoting endophyte Variovorax paradoxus S110. J Bacteriol. 2011;193(5):1183-1190

\section{2. !!! INVALID CITATION !!!}

33. Stępniewska Z, Kuźniar A. Endophytic microorganisms-promising applications in bioremediation of greenhouse gases. Applied Microbiology and Biotechnology. 2013;97(22):9589-9596.

34. Weyens N. Phytoremediation:plant-endophyte partnerships take the challenge. Current Opinion in Biotechnology. 2009;20(2):248-254.

35. Rajkumar M, Ae N, Freitas H. Endophytic bacteria and their potential to enhance heavy metal phytoextraction. Chemosphere. 2009;77(2):153160 .
36. Radwan S. Phytoremediation for Oily Desert Soils, in Advances in Applied Bioremediation, Singh A, Kuhad CR, and P.O. Ward, Berlin, Heidelberg: Editors Springer Berlin Heidelberg; 2009. p. 279-298.

37. Olson PE. Effects of agronomic practices on phytoremediation of an aged PAH-contaminated soil. J Environ Qual. 2008;37(4):1439-1446.

38. A1-Mailem D. Oil phytoremediation potential of hypersaline coasts of the Arabian Gulf using rhizosphere technology. Bioresource technology. 2010;101(15):5786-5792.

39. Rybakova D. Endophytes-assisted biocontrol: novel insights in ecology and the mode of action of Paenibacillus. Plant and Soil. 2015. p. 1-16.

40. Gao F, Dai C, Liu X. Mechanisms of fungal endophytes in plant protection against pathogens. Afr J Microbiol Res. 2010;4(13):1346-1351.

41. Castillo U. Kakadumycins, novel antibiotics from Streptomyces sp NRRL 30566, an endophyte of Grevillea pteridifolia. FEMS Microbiol Lett. 2003; 224(2):183-190.

42. Christina A, Christapher V, Bhore SJ. Endophytic bacteria as a source of novel antibiotics: sAn overview. Pharmacognosy Reviews 2013;7(13):11-16

43. Perring TM, Gruenhagen NM, Farrar CA. Management of plant viral diseases through chemical control of insect vectors. Annual review of entomology. 1999;44(1): 457-481.

44. Hayat R. Soil beneficial bacteria and their role in plant growth promotion:a review. Annals of Microbiology. 2010;60(4):579-598.

45. Mei C, Flinn BS. The use of beneficial microbial endophytes for plant biomass and stress tolerance improvement. Recent Pat Biotechnol. 2010;4(1):81-95.

46. Schulz B, Boyle C. What are Endophytes? in Microbial Root Endophytes, PDDBJE Schulz, et al. editors. 2006, Springer Berlin Heidelberg; 2006. p. 1-13.

47. Hartley SE, Gange AC. Impacts of plant symbiotic fungi on insect herbivores: mutualism in a multitrophic context. Annu Rev Entomol. 2009;54:323-342.

48. Clay K, Schardl C. Evolutionary origins and ecological consequences of endophyte symbiosis with grasses. Am Nat. 2002;Suppl 160(4):99-S127.

49. Benhamou N, Kloepper JW, Quadt-Hallman A, et al. Induction of defense-related ultrastructural modifications in pea root tissues inoculated with endophytic bacteria. Plant Physiol. 1996;112(3):919-929.

50. Harish S, Kavino M, Kumar N, et al. Induction of defense-related proteins by mixtures of plant growth promoting endophytic bacteria against Banana bunchy top virus. Biological Control. 2009;5(1):16-25.

51. Zehnder GW, Changbin Yao, John F Murphy, et al. Induction of resistance in tomato against cucumber mosaic cucumovirus by plant growthpromoting rhizobacteria. Biocontrol. 2000;45(1):127-137.

52. Yue Q, Chunlin Wang, Thomas J Gianfagna, et al. Volatile compounds of endophyte-free and infected tall fescue (Festuca arundinacea Schreb.). Phytochemistry. 2001;58(6):935-941.

53. Quadt-Hallmann A, Kloepper JW, Benhamou N. Bacterial endophytes in cotton:mechanisms of entering the plant. Canadian journal of microbiology. 1997;43(6):577-582.

54. Piccoli P, Bottini R. Abiotic stress tolerance induced by endophytic PGPR, in Symbiotic Endophytes. Springer; 2013. p. 151-163.

55. Fernandez O, Theocharis A, Bordiec S, et al. Burkholderia phytofirmans PsJN acclimates grapevine to cold by modulating carbohydrate metabolism. Molecular Plant-Microbe Interactions. 2012;25(4):496-504. 
56. Barka EA, Nowak J, Clément C. Enhancement of chilling resistance of inoculated grapevine plantlets with a plant growth-promoting rhizobacterium, Burkholderia phytofirmans strain PsJN. Applied and environmental microbiology. 2006;72(11):7246-7252.

57. Naveed M, Baqir Hussain M, Zahir A Zahir, et al. Drought stress amelioration in wheat through inoculation with Burkholderia phytofirmans strain PsJN. Plant growth regulation. 2014;73(2):121-131.

58. Jha Y, Subramanian R, Patel S. Combination of endophytic and rhizospheric plant growth promoting rhizobacteria in Oryza sativa shows higher accumulation of osmoprotectant against saline stress. Acta physiologiae plantarum. 2011;33(3):797-802.

59. Zhang S, Outlaw W. Abscisic acid introduced into the transpiration stream accumulates in the guard-cell apoplast and causes stomatal closure. Plant, Cell \& Environment. 2001;24(10):1045-1054.

60. Feng J, Barker AV. Ethylene evolution and ammonium accumulation by tomato plants under water and salinity stresses. Part II. Journal of plant nutrition. 1992;15(11):2471-2490.
61. Nadeem SM. Microbial ACC-deaminase:prospects and applications for inducing salt tolerance in plants. Critical reviews in plant sciences. 2010;29(6):360-393.

62. Aschehoug ET. Fungal endophytes directly increase the competitive effects of an invasive forb. Ecology. 2012;93(1):3-8.

63. Kuldau G, Bacon C. Clavicipitaceous endophytes:their ability to enhance resistance of grasses to multiple stresses. Biological Control. 2008;46(1):57-71.

64. Malinowski DP, Alloush GA, Belesky DP. Leaf endophyte Neotyphodium coenophialum modifies mineral uptake in tall fescue. Plant and Soil. 2000;227(1-2):115-126.

65. Ali S, Charles TC, Glick BR. Amelioration of high salinity stress damage by plant growth-promoting bacterial endophytes that contain ACC deaminase. Plant Physiol Biochem. 2014;80:160-167.

66. Bacon CW, Hinton DM. Bacterial endophytes:The endophytic niche, its occupants, and its utility, in Plant-Associated Bacteria, S.S. Gnanamanickam, Editor. Dordrecht: Springer Netherlands; 2006. p. 155-194. 